\title{
Left Ventricle Statistical Models Segmentation of Shape and Appearance for Analysis of Cardiac MRI
}

\author{
Kayte Jaypalsing Natthusing ${ }^{1}$ \\ Computer Science Dept. of CS \& \\ IT, Dr. Babasaheb Ambedkar \\ Marathwada University, \\ Aurangabad(MH), India.
}

\author{
Sumegh Shrikant Tharewal ${ }^{2}$ \\ BSR Research Fellow, Dept. of CS \\ \& IT, Dr. Babasaheb Ambedkar \\ Marathwada University, \\ Aurangabad(MH), India.
}

\author{
Mohammed Waseem \\ Ashfaque $^{3}$ \\ Dept of CS and IT, \\ Dr. Babasheb Ambedkar \\ Marathwada University, \\ Aurangabad $(\mathrm{MH})$ India,
}

\author{
Manza Ramesh Raybhan ${ }^{6}$, \\ $\mathrm{PhD}$ \\ Assistant Professor of Computer \\ Science Dept. of CS \& IT, Dr. \\ Babasaheb Ambedkar Marathwada \\ University, Aurangabad (MH), India.
}

\begin{abstract}
This paper proposes a design of a framework structure for analysis of cardiac MRI to find out cardiovascular Disease easily and increase patent life. Segmentation of volumetric medical data is extremely time- consuming if using semiautomatically segmentation techniques with the first contribution involves the introduction of a new algorithm for fitting 4D Active Appearance Models on cardiac MRI, using the Simple interactive object extraction (SIOX), have observe a 43- fold increase in fitting accuracy that is on par with fuzzy clustering. We show the high quality results that are derived by the use of fuzzy clustering, and describe the ways in which it could improve the automated analysis of medical images.
\end{abstract}

Keywords: MRI, SIOX, Fuzzy Clustering,

\section{INTRODUCTION}

In 2010, Cardiovascular Disease (CVD) contributed to almost one third of global deaths. CVD is the leading cause of death in the developed world and by 2013; CVD is estimated to be the main cause of death in developing countries. According to 2001 estimates, if all forms of CVD in the India were eliminated, the average life expectancy would increase by around ten years. An elimination of all forms of cancer, on the other hand, would cause the average life expectancy to increase by three years [2].

Three dimensional imaging of the heart using imaging modalities such as Ultrasound, Magnetic Resonance Imaging (MRI) and X-ray Computed Tomography is a rapidly developing area of research in medical imaging. Screenings that detect problems at an early stage, when treatment is most effective, can help prevent heart disease. The manual segmentation of short axis cardiac MRI (identifying the various structures of interest in the image) can provide clinically.
Recently a lot of extensions for AAMs have been proposed. While the original AAMs work with gray value images in 2D, different strategies for adaptations to higher dimensions have been suggested. In theory such adaptations can be done straight forward. Practically there are some critical aspects. Different methods have been proposed to overcome the problems in higher dimensions. Applications include timecontinuous (2D+time) segmentation of image sequences [Lelieveldt et al., 2001; Edwards et al., 1998], real-time combined 2D+3D AAMs [Xiao et al. 2004], and bi-temporal 3D AAMs [Stegmann and Pedersen, 2005]. Normally an increase in dimensionality inevitably causes a rapid increase of data. Especially the number of texture samples mounts significantly for higher dimensional data. Large data is the reason why methods for compression of texture data using wavelets [Wolstenholme and Taylor, 1999; Stegmann et al., 2004] or wedgelets [Darkner et al., 2004] recently have been proposed [2].

The Standard numerical optimization methods for the fitting of AAMs, such as gradient descent, are very slow, mainly due to the high number of parameters that have to be optimized. when moving from 2-D AAMs to 3-D AAMs this problem is further exacerbated. When using 3-D AAMs for the segmentation of the left ventricle, it is not uncommon for such models to use 50-100 parameters. an effort to deal with this, efficient algorithms for fitting AAMs have been developed. However, the fitting accuracy and the convergence rates of such algorithms are known to be suboptimal in many applications. Recently, a novel algorithm for the fitting of 2-D AAMs was introduced in. Its applicability was demonstrated on artificial data and on real life data for face tracking. However, as cites, there was no known way of extending the algorithm to higher dimensions. This was mainly so because a certain argument used in the paper applied only to 2-D similarity transformations. This literature present an extension 
of this algorithm for the fitting of 3-D AAMs, when used for the segmentation of short axis cardiac MRI. By definition, short axis cardiac MR images are such that the long axis of the heart is perpendicular to the acquisition image plane. In practice, this means that during the AAM fitting we need to rotate our model only around the long axis of the heart. We take advantage of this fact to design an efficient fitting algorithm, which will rotate the model about the axis perpendicular to the image acquisition plane. To the best of our knowledge, this is the first effort at extending the inverse compositional image alignment algorithm to 3-D AAMs, and testing its applicability to the interpretation of medical images.

The algorithms described in the literature for fitting AAMs, can be classified as either robust but inefficient gradient descent type algorithms, or as the efficient but ad-hoc algorithms described next. The original AAM formulation uses regression to find a matrix $\mathrm{R}$, such that if the current fitting error between the AAM and the image is $\delta$, the updated AAM parameters are $\delta \mathrm{p}=\mathrm{R} \delta \mathrm{t}$. In more recent implementations, the estimation of matrix $\mathrm{R}$ is superseded by a faster and simpler method which regards $\mathrm{R}$ as a Jacobian matrix of the error function between the AAM and the image. In general, there is no reason why the error measure $\delta$ t should uniquely identify the update parameters $\delta p$. Such methods lack a sound theoretical basis. Moreover, it has been shown that using a matrix $\mathrm{R}$ to estimate the update parameters can lead to suboptimal results. However, the constant matrix technique is widely used due to its fitting speed. Later, in, it was shown how to use the inverse compositional image alignment algorithm to fit 2-D AAMs.

The algorithm we describe in this paper is an extension of 3$\mathrm{D}$, under the constraint that all rotations take place around one axis. Our experimental results show that our algorithms border positioning errors are significantly smaller than the errors reported for other 3-D AAMs which use the constant matrix approach for the fitting. We perform experiments comparing our algorithm with Gauss-Newton based optimization, which is generally known as one of the most accurate and reliable optimization algorithms for such problems. We observe a 60 -fold improvement in the fitting speed, with a segmentation accuracy that is as good - and in many cases better - as brute force Gauss-Newton optimization. We did not use any hierarchical coarse-to-fine methods during the optimization, to speed up the fitting process, however the effects of such an approach on the fitting algorithms could be a topic of future research

This is an example of the basic Active Shape Model (ASM) and also the Active Appearance Model (AAM) as introduced by Cootes and Taylor, 2D and 3D with multi-resolution approach, color image support and improved edge finding method. Very useful for automatic segmentation and recognition of biomedical objects[2].

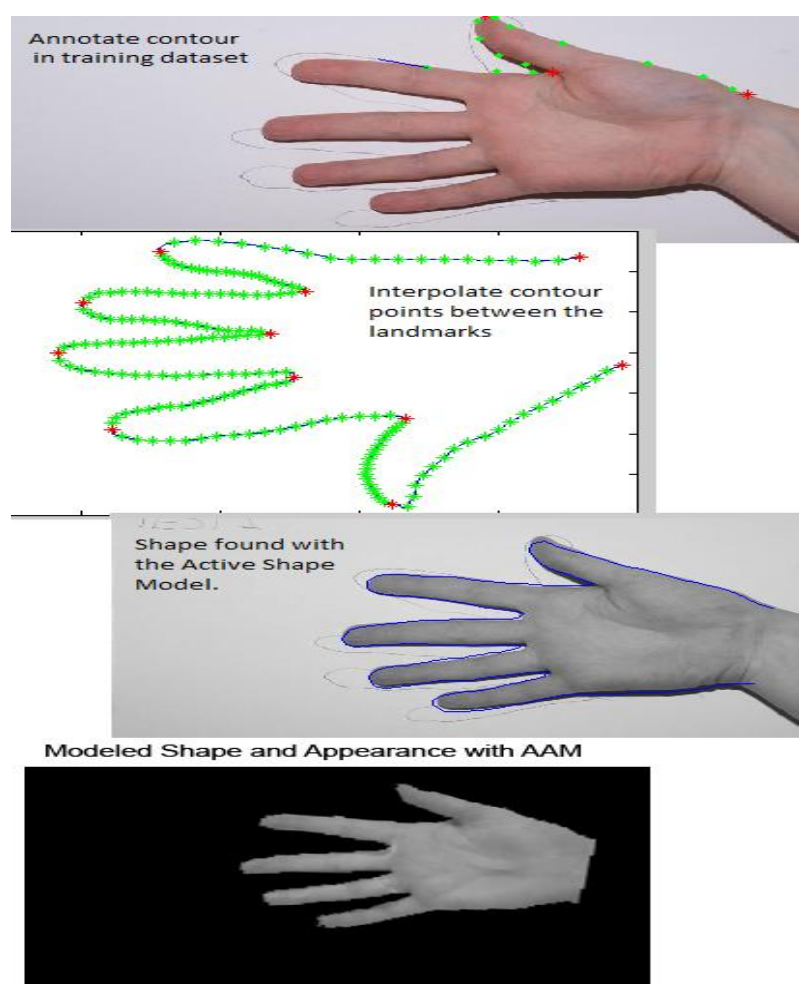

Figure 1. Hand Shape Appearance Model [4].

\subsection{Functional Anatomy of Coronary Vessels}

The right and left coronary arteries arise at the root of the aorta behind the right and left cusps of the aortic valve, respectively. These arteries provide the entire blood supply to the myocardium. The right coronary artery principally supplies the right ventricle and atrium. The left coronary artery, which divides near its origin into the anterior descending and the circumflex branches, mainly supplies the left ventricle and atrium. There is some overlap between the regions supplied by the left and right arteries. In humans, the right coronary artery is dominant (supplying most of the myocardium) in about $50 \%$ of individuals. The left coronary artery is dominant in another $20 \%$, and the flow delivered by each main artery is about equal in the remaining $30 \%$. The epicardial distribution of the coronary arteries and veins is illustrated in Figure 2[13]. 

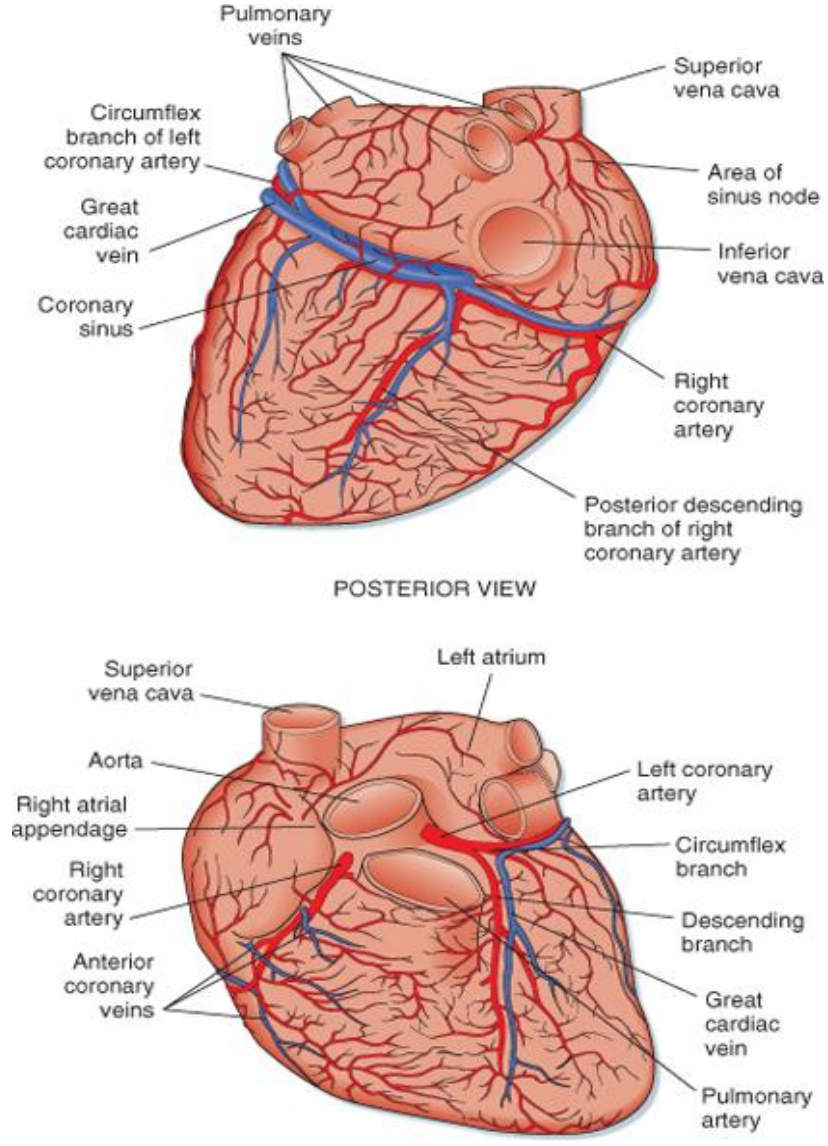

ANTEPIOR VIEW

Koeppen \& Stanton: Berne and Levy Physiology, 6th Edition.

Copyright (C) 2008 by Mosby, an imprint of Elsevier, Inc. All rights reserved

Figure 2. An Anterior and posterior surfaces of the heart illustrating thelocation and distribution of the principal coronary vessels13.

Coronary arterial blood passes through the capillary beds; most of it returns to the right atrium through the coronary sinus. Some of the coronary venous blood reaches the right atrium via the anterior coronary veins. In addition, vascular communications directly link the myocardial vessels with the cardiac chambers; these communications are the arteriosinusoidal, arterioluminal, and thebesian vessels. The arteriosinusoidal channels consist of small arteries or arterioles that lose their arterial structure as they penetrate the chamber walls, where they divide into irregular, endotheliumlined sinuses. These sinuses anastomose with other sinuses and with capillaries, and they communicate with the cardiac chambers. The arterioluminal vessels are small arteries or arterioles that open directly into the atria and ventricles. The the besian vessels are small veins that connect capillary beds directly with the cardiac chambers and also communicate with the cardiac veins. All the minute vessels of the myocardium communicate in the form of an extensive plexus of subendocardial vessels. However, the myocardium does not receive significant nutritional blood flow directly from the cardiac chambers [13].

\subsection{Basic idea ASM}

The ASM model is trained from manually drawn contours (surfaces in 3D) in training images. The ASM model finds the main variations in the training data using Principal Component Analysis (PCA), which enables the model to automatically recognize if a contour is a possible/good object contour. Also the ASM modes contains matrices describing the texture of the lines perpendicular to the control point, these are used to correct the positions in the search step.

After creating the ASM model, an initial contour is deformed by finding the best texture match for the control points. This is an iterative process, in which the movement of the control points is limited by what the ASM model recognizes from the training data as a "normal" object contour.

\subsection{Basic idea AAM}

PCA is used to find the mean shape and main variations of the training data to the mean shape. After finding the Shape Model, all training data objects are deformed to the main shape, and the pixels converted to vectors. Then PCA is used to find the mean appearance (intensities), and variances of the appearance in the training set. Both the Shape and Appearance Model are combined with PCA to one AAMmodel.

By displacing the parameters in the training set with a know amount, and model can be created which gives the optimal parameter update for a certain difference in model-intensities and normal image intensities. This model is used in the search stage.

\section{PROCESS OF DATA ANALYSIS IN SIMPLE FORM}

\subsection{Image Analysis}

Image analysis is the process of extracting meaningful information from images such as finding shapes, counting objects, identifying colors, or measuring object properties.

Image Processing Toolbox provides a comprehensive suite of reference-standard algorithms and visualization functions for image analysis tasks such as statistical analysis, feature extraction, and property measurement.

\subsection{Image Transforms}

Image transforms play a critical role in many image processing tasks, including image enhancement, analysis, restoration, and compression. Image Processing Toolbox provides several image transforms, including Hough, Radon, FFT, DCT, and fan-beam projections. You can reconstruct images from parallel-beam and fan-beam projection data (common in tomography applications).

\subsection{Hough Transform}

The Hough transform is designed to identify lines and curves within an image. Using the Hough transform you can:

- Find line segments and endpoints

- Measure angles

- Find circles based on size

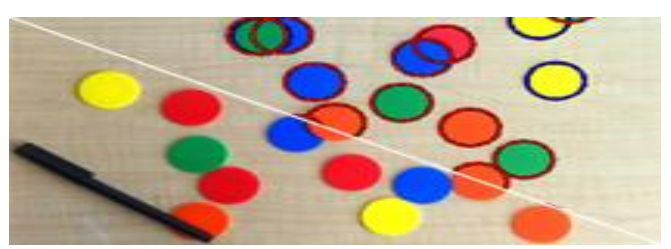

Figure 3. Detect and Measure Circular Objects in an Image Statistical Functions 
2.4 Statistical functions let you analyze the general characteristics of an image by:

- Computing the mean or standard deviation

- Determining the intensity values along a line segment

- Displaying an image histogram

- $\quad$ Plotting a profile of intensity values

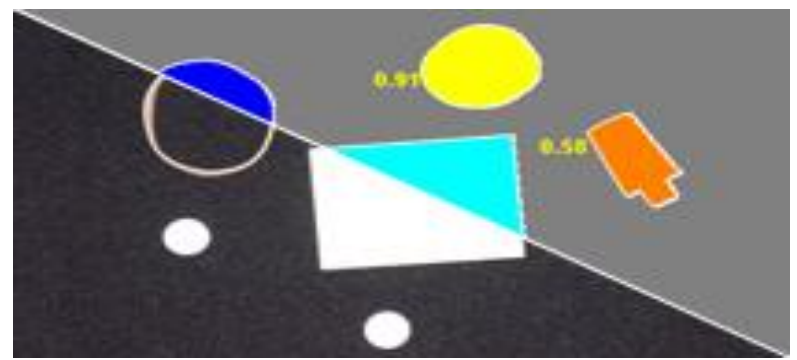

Figure 3.1 Identifying Round Objects Device-Independent Color Management

Device-independent color management enables you to accurately represent color independently from input and output devices. This is useful when analyzing the characteristics of a device, quantitatively measuring color accuracy, or developing algorithms for several different devices. With specialized functions in the toolbox, you can convert images between device-independent color spaces, such as $\mathrm{sRGB}, \mathrm{XYZ}$, xyY, L*a*b*, uvL, and L*ch.
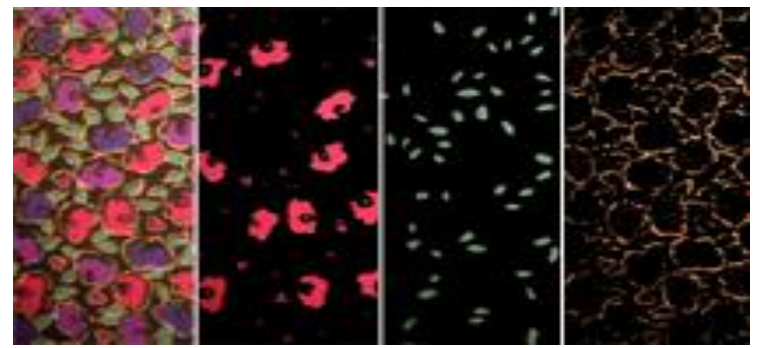

Figure 3.2 Color-Based Segmentation Using the $L^{*} a^{*} b^{*}$ Color Space

\section{METHODS}

\subsection{Optimization of 3D AAMs for short axis} cardiac MRI segmentation

Active appearance models (AAMs) provide a robust approach for the analysis of medical images (Cootes and Taylor, 1998, 2004). The ability of AAMs to learn the 3D structure of the heart and not lead to unlikely segmentations has stirred up interest in the medical imaging community regarding their use for the segmentation of the left ventricle from short axis cardiac MRI (Frangi et al., 2001; Mitchell et al., 2002).

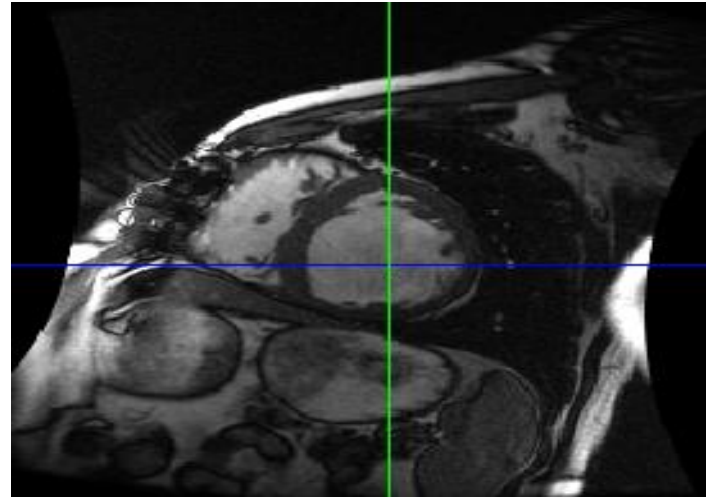

Figure4 (A): Short axis cardiac MRI.

\subsection{3-D. Active appearance Model (AAMs)}

In this section, we describe our implementation of the 3-D AAM of the left ventricle and its application for cardiac MRI segmentation. It has some similarities to the methodology used in but with many novel differences. We begin by quickly over-viewing point distribution models (PDMs) for 3-D AAMs. We proceed by describing how we align the landmarks which made up our training set and we conclude with an overview of how we handle appearance variation.
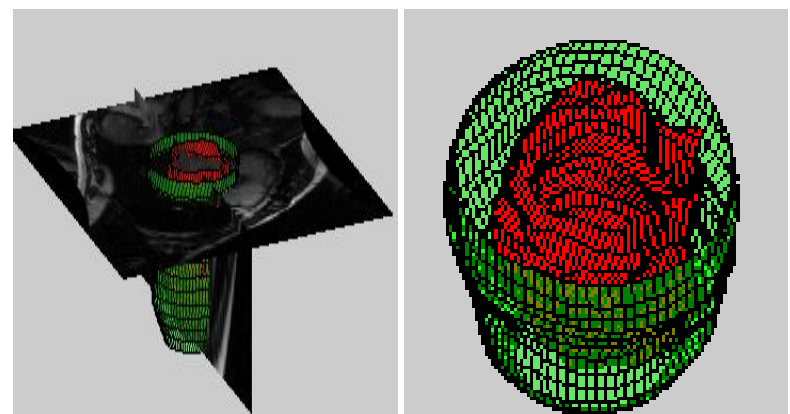

Figure 4 (B): Endocardial and epicardial landmarks stacked on top of each other. displayed as curves for greater clarity.

\subsection{The Point Distribution Model}

Figure 4(B) represents a short axis cardiac MR image. A stack of such images gives us a volumetric representation of the heart. Manual segmentations of the left ventricle provide contours representing the endocardium and epicardium of the left ventricle. By uniformly sampling each of these contours at i0 points along their arc length, each contour is represented by a set of landmarks. In our implementation each slice consists of the same number of landmarks. By stacking the landmarks on top of each other we obtain a 3-D representation of the endocardium and epicardium of the left ventricle, as shown on figure 1.B. However, the number of images intersecting the left ventricle is not the same for every patient. Therefore, we need to interpolate between the contours so that every 3-D model is made up of the same number of slices. If we want to create a contour at height $\mathrm{zO}$ located between two contours, we can simply do the following: From the line segment joining the landmark in the two contours, we find the location with height $\mathrm{z} 0$. This gives the landmark in the new contor. In our implementation, we created 15 contours, evenly sampled along the z-axis, located between the apex and basal contours, as shown in figure 4.B. 
Assuming that we have a set of $\mathrm{N}$ sample shapes, each sample made up of 1 landmarks, we can represent each shape sample as a 31 dimensional vector, since each landmark is made up of 3 coordinates. Applying principal component analysis (PCA) on the distribution of the shape vectors, any shape s out of the $\mathrm{N}$ shapes can be represented as

$$
\mathrm{s} \approx \mathrm{s}_{0}+\sum_{i=1}^{n} p_{i} \mathrm{~s}_{i}
$$

For some $\mathrm{p}=(\mathrm{p} 1, \ldots, \mathrm{pn}) \in \_\mathrm{n}$, where $\mathrm{s} 0$ is the mean shape vector (a.k.a base mesh), and si indicates the ith eigenvector. We are summing over $n$ eigenvectors si with eigenvalues $\lambda 1 \geq$ $\lambda 2 \geq \ldots \geq \lambda \mathrm{n} \geq 0$. These are the $\mathrm{n}$ eigenvectors with highest eigenvalues that we found with PCA. We choose a value for $n$ such that it accounts for around $90 \%-95 \%$ of the variation. Empirically, it has been hown that this is a good value. Higher values tend to lead to PDMs which overfit the training set, and much smaller values lead to PDMs which cannot generalize to new shapes. The greater the value of $n$, the better the approximation in becomes.

\subsection{Appearance Variation}

Here need to model the appearance variation of the volume enclosed by the shape. Firstly, we manually tetrahedrize s0, as shown in figure 4.C. This splits the left ventricular volume enclosed by $\mathrm{s} 0$ into tetrahedra whose appearance we model. In other words we are modeling the appearance of the interior of the LV (in the same way that Mitchell did in), and not just the appearance of the walls of the endocardium and epicardium. We use the same landmark connectivity defining the tetrahedra of s0 to define the tetrahedrization of any shape variation resulting from Equation above. Then, we sample the appearance enclosed by each training shape using the same methodology as in which is a $3 \mathrm{D}$ extension of the method described it for the 2D case: Firstly, we choose a set of sampling points inside each tetrahedron of s0. Each such point has a barycentric coordinate with respect to the tetrahedron enclosing it (by definition, the summation of the barycentric coordinates must equal 1). Then, we sample each tetrahedron in the training set at the same barycentric coordinates that we sampled its corresponding tetrahedron in $\mathrm{s} 0$. We review in more detail the definition of barycentric coordinates, and how to sample the interior of each tetrahedron.



Figure 4(c): Tetrahedrization of base mesh s0. Every tetrahedron represents a part of the myocardial muscle or a part of the left ventricle's blood pool [1].

Let the mean appearance we get by averaging the sampled appearances be $\mathrm{A} 0(\mathrm{x})$ and the $\mathrm{k}$ eigenvectors we found by PCA, describing around $90 \%-95 \%$ of the appearance variation (defined in section 3.1), be A1(x), A2(x), .., Ak(x) (where $\mathrm{x}$ denotes a voxel coordinate where we sampled the base model s0). Then for different values of the bi

$$
A(\mathrm{x})=A_{0}(\mathrm{x})+\sum_{i=1}^{k} b_{i} A_{i}(\mathrm{x})
$$

Defines the different appearance variations the model has learned from our training data.

\section{QUALITATIVE RESULTS}

Above we have considered models which were matched to all data sets. In this section we concentrate on more details of individual AAM searches applied to selected single data sets. The intention is to show how different parameters influence the matching process.

\subsection{Leave-One-Out}

From the quantitative results it can be learned that data set number 13 leads to better matching results than data set number 18. The reason for this difference in quality of matches seems to be that data set 13 explains its appearance by modes that represent statistically frequent details. Data set 18 on the other hand seems to comprise statistically rare features.

Both data sets 13 and 18 were taken from the set of 15 data sets manually identified as qualitatively good. A leave-oneout test was carried out such that for both data sets a model of the remaining 14 data sets was built and then matched with the one that was left out.

For both data sets multiple AAM searches were performed which differ in the initial displacements of the model's position. Figure 5. shows the progress of matching in terms of APS. For all initial positions the model converges on data set 13. The matching of data set 18 proceeds not so stable and diverges for two of the four tests.

In the following we present results of matching the model from 14 good data sets to data set 13. Figure5.1. the model initially was displaced by $15 \mathrm{~mm}$ in direction of the $\mathrm{X}$ - and by $30 \mathrm{~mm}$ in direction of the $\mathrm{Y}$-axis. Red color represents a point to- surface distance of $10 \mathrm{~mm}$ or more. Blue indicates a pointto-surface distance of $5 \mathrm{~mm}$ and green a distance of $0 \mathrm{~mm}$. Other color values are interpolated accordingly. The color coded surface distance is only calculated for individual model points and not over the whole surface. Colors are smoothly interpolated between points in the mesh. The black wire frame represents the shape of the ground truth for the considered data set 13 .

Figure 5.1 shows the converged model together with the ground truth. Endoand epicardium of model and ground truth are shown separately.

Figure5.3. illustrates the differences in texture of the same matching process using volume visualization. The three volumes shown are again the difference volumes before the first, after the 4th and after the 8th (converged) iteration. The same matching process is visualized differently in figure 5.4. Three slices were interpolated for the initial model placement, after the 4th iteration and after the 8th (converged) iteration. The data together with the model superimposed on it are shown. The bottom row shows the original data with the shape of the converged model. 

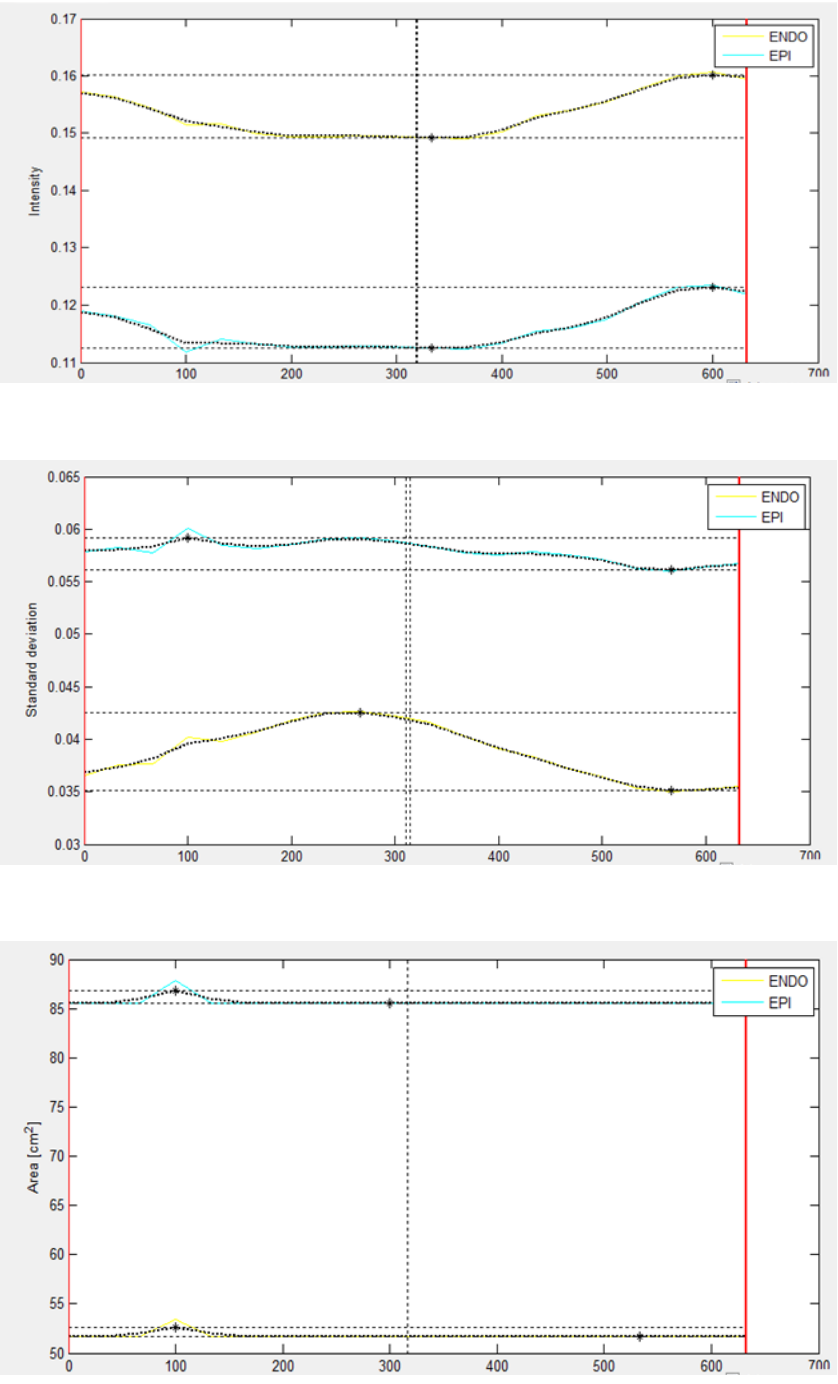

Figure 5: progress of show result area, Standard Deviation, or intensity of ENDO / EPI the cardiac MRI.

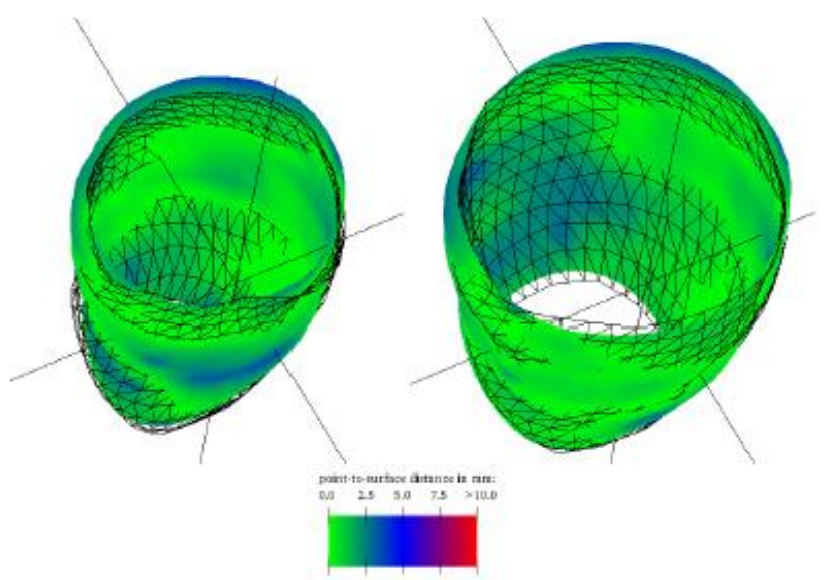

Figure 5.1: Matching data set 13. The Result is shown for endocardium (left) and epicardium (right) separately[3].

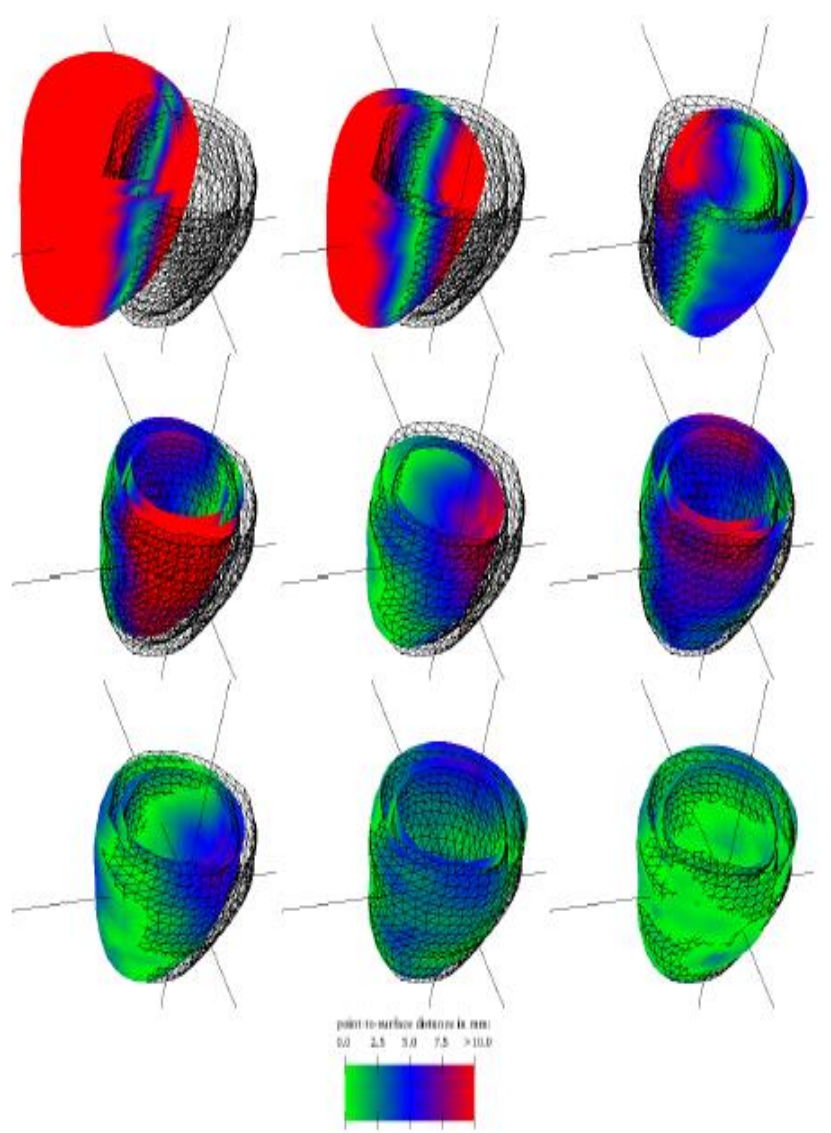

Figure 5.2: Matching data set 13 (all iterations) with a model built from 14 data sets not including data set 13 . The matching process starts at the image on the top left and ends at the right bottom. Each image represents one iteration. [3]

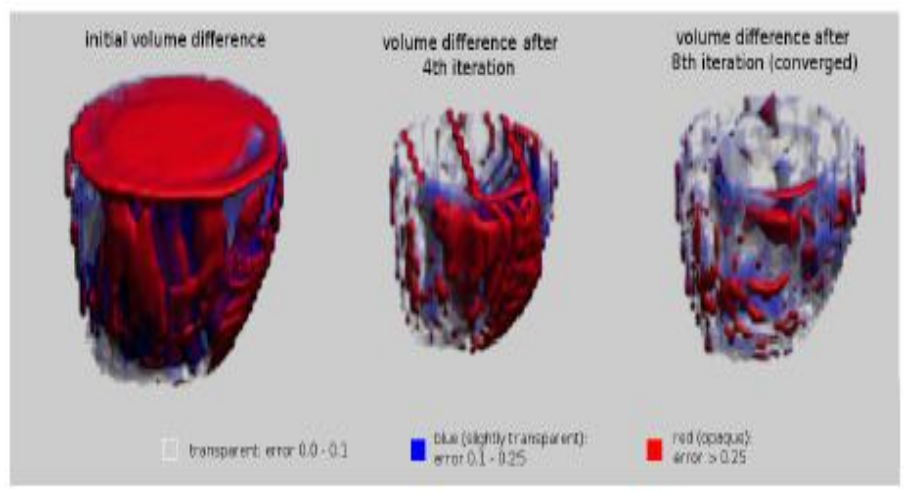

Figure 5.3: Matching data set 13 (difference volumes).

This figure shows the initial difference volume, the difference volume after 4th, and after 8th (converged) iteration [3]. 

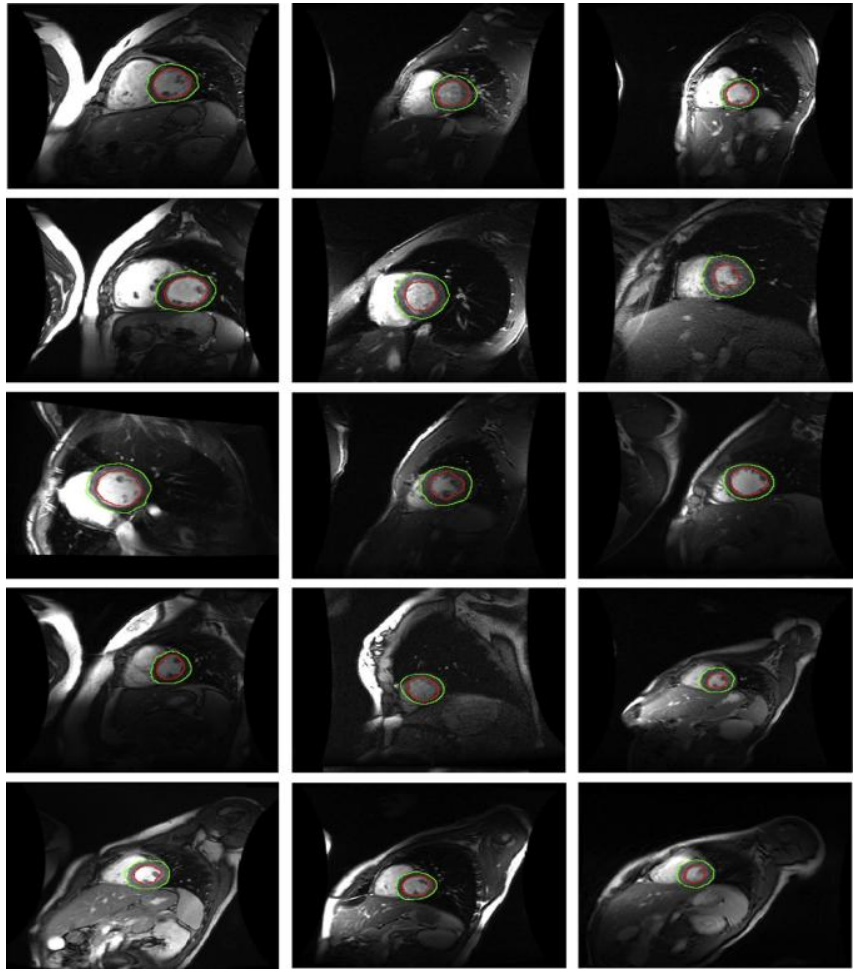

Figure 5.4: Matching data set 13 with slice-wise texture visualization. The top row shows three slices of data with the initial model superimposed. The second and third rows show the model after the 4th and 8th (converged) iteration respectively. The bottom row shows the data with the matched model's shape points.

\section{CONCLUSION}

In this paper we have discussed 4D cardiac MRI data. We first presented an algorithm for fitting 4D active appearance models on short axis cardiac MR images, and observed an almost 43-fold improvement in the segmentation speed and a segmentation accuracy that is on par (and often better) with Simple interactive object extraction (SIOX), the most widely used algorithm for such optimization problems. We have outlined the importance of fast automatic and semiautomatic segmentation of such data. We shortly reviewed the anatomical background and outlined special properties of cardiac MRI data.

\section{REFERENCES}

[1] Medical Image Analysis 12 (2008) 335-357 Alexander Andreopoulos, John k. Tsotsos, York University, Dept of Computer science and Engineering, center for vision Research, Toronto, Ontario, Canada M3J 1P3

[2] American Heart Association, 2004. International Cardiovascular Disease Statistics. http://www.americanheart.org> (Online).

[3] 3D Active Appearance Models for Segmentation of Cardiac MRI Data durch Sebastian Zambal Matr. Nr.: 9826978 A - 3340 Waidhofen/Ybbs, Ybbsitzerstr. 44a.

[4] Automatic Segmentation of Contrast Enhanced Cardiac MRI for Myocardial Perfusion Analysis durch Andreas ch"ollhuber Matr. Nr.: 0226055 A - 1040 Wien, Wiedner G"urtel 24

[5] Manuel D. Cerqueira, Neil J.Weissman, Vasken Dilsizian, Alice K. Jacobs, Sanjiv Kaul, Warren K. Laskey, Dudley J. Pennell, John A. Rumberger, Thomas Ryan, and Mario S. Verani. Standardized myocardial segmentation and nomenclature for tomographic imaging of the heart. Circulation, 105(4):539-42, 2002.

[6] Baker, S., Matthews, I., 2001. Equivalence and efficiency of image alignment algorithms. In: Proceeding of the IEEE Conference on Computer Vision and Pattern Recognition, vol. 1, pp. 1090-1097.

[7] Baker, S., Goss, R., Matthews, I., 2004. Lucas-Kanade 20 years on: a unifying framework. Int. J. Comput. Vis. 56 (3), 221-255.

[8] Bardinet, E., Ayache, N., Cohen, L.D., 1996. Tracking and motion analysis of the left ventricle with deformable superquadrics. Med. Image Anal. 1 (2), 129-150.

[9] Papademetris, X., Sinusas, A., Dione, D., Constable, R., Duncan, J., 2002.Estimation of 3D left ventricular deformation from medical imagesusing biomechanical models. IEEE Trans. Med. Imaging 21 (7), 786-800.

[10] Davatzikos, C., Tao, X., Shen, D., 2003. Hierarchical active shape models, using the wavelet transform. IEEE Trans. Med. Imaging 22 (3), 414-422.

[11] Hamarneh, G., Gustavsson, T., 2004. Deformable spatiotemporal shape models: extending ASM to $2 \mathrm{D}+$ time. Image Vis. Comput. 22 (6),461-470. 